\title{
Estimation of VaR Using Copula and Extreme Value Theory
}

\author{
L. K. Hotta \\ State University of Campinas, Brazil \\ E. C. Lucas \\ ESAMC, Brazil \\ H. P. Palaro \\ State University of Campinas, Brazil and \\ Cass Business School, U.K.
}

This paper proposes a method for estimating the VaR of a portfolio based on copula and extreme value theory. Each return is modeled by ARMAGARCH models with the joint distribution of innovations modeled by copula. The marginal distributions are modeled by the generalized Pareto distribution in the left tail (large loss) and empirical distribution otherwise. The copula is estimated by an estimator which gives more weight to observations with large loss. The method is applied to a two-asset portfolio and compared to other traditional methods (JEL: C15, D81,G10).

Keywords: conditional copula, risk measures, VaR, extreme value theory.

\section{Introduction}

Value at Risk (VaR) is largely used to measure the risk of a portfolio. One of the main difficulties in estimating $\mathrm{VaR}$ is to model the dependence structure, especially because $\mathrm{VaR}$ is concerned with the tail of the distribution. This paper proposes a model based on a dynamic econometric model for volatility, Copula Theory and Extreme Value Theory (EVT). ARMA-GARCH models are fitted to each return in order to explain the level and the (conditional) volatility of returns. The heteroskedasticity-adjusted observations are modeled by copula models whereas the marginal distributions are modeled by empirical distribution or by the generalized Pareto distribution (GPD) in the left tail (large loss)

* The authors acknowledge financial support from CNPq, PRONEX and FAPESP.

(Multinational Finance Journal, 2008, vol. 12, no. 3/4, pp. 205-218)

Quarterly publication of the Multinational Finance Society, a nonprofit corporation.

(C) Global Business Publications. All rights reserved.

DOI: $10.17578 / 12-3 / 4-3$ 
and by empirical distribution otherwise. An estimator, which gives more weight to observations with larger relative losses, is proposed to estimate the copula. The main contributions of the paper are the proposed marginal copula distributions and the estimation procedure. The estimation procedure allows modeling data with asymmetric dependence with copula families with symmetric dependence.

The paper is organized as follows. Section II introduces the portfolio model. Section III presents the main features of copula models as the relate to portfolio VaR estimation. Section IV presents an application to a portfolio of Brazilian and Argentinean stock market indices and compares the performance of the copula models to those of traditional univariate and bivariate methods. Section V concludes.

\section{The Portfolio Model}

Consider a portfolio composed of $n$ assets. Let $r_{j, t}$ the log returns of the $j^{\text {th }}$ asset at time $t$. Each return is modeled by an ARMA-GARCH model. For example the $\mathrm{AR}(1)-\mathrm{GARCH}(1,1)$ model is specified as:

$$
\begin{gathered}
r_{j, t}=c_{j}+\varphi_{j} r_{j, t-1}+\varepsilon_{j, t} \\
\varepsilon_{j, t}+\sigma_{j, t} \eta_{j, t} \\
\sigma_{j, t}^{2}=\alpha_{j}+\beta_{j} \varepsilon_{j, t-i}^{2}+\gamma_{j} \sigma_{j, t-i}^{2},
\end{gathered}
$$

where the innovations $\left\{\eta_{j, t}\right\}$ are white noise processes with zero mean and unit variance, $\alpha_{j}, \beta_{j}$ and $\gamma_{j}$ follow the Nelson and Cao (1992) restrictions, $\beta_{j}+\gamma_{j}<1$ and $j=1, \ldots, n$ and $t=1, \ldots, T$. The distribution of the random vector $\eta_{j}=\left(\eta_{1}, \ldots, \eta_{n, t}\right)$ is modeled by copula, e.g., Dias and Embrechts (2004) and Patton (2006b). The ARMA-GARCH models work as a filter in order to obtain an innovation processes, i.e., a serially independent process. Di Clemente and Romano (2005) use a variance-covariance matrix estimated using exponentially weighted moving averages to filter the data. All of them report some evidence that the random vector $\eta_{t}$ is not normally distributed. The multivariate $t$ distribution is also not adequate because the condition that all marginal distributions should have the same degree of freedom is too restrictive. Decomposing the multivariate distribution into the marginal distributions, the copula seems to be a natural alternative because it allows modeling individual variables and enlarging the family of multivariate distributions. In this work, the marginal are modeled by two distributions: by the empirical distribution 
and by the GPD distribution in the lower tail and by the empirical distribution otherwise. The use of extreme value distribution is particularly important for emerging markets where it is expected to have heavy tails. For example, Seymour and Polakow (2003) use univariate extreme value theory to model a portfolio of South African stocks.

\section{A. Value at Risk (VaR)}

$\mathrm{VaR}$ has become the standard measure used by financial analysts to quantify the market risk of an asset or a portfolio. Although the great popularity of this instrument lies in the simplicity of interpretation, its estimation is a non-trivial issue. Manganelli and Engle (2004) present a survey of different approaches, their underlying assumptions and their pros and cons. However, they draw attention to the fact that the number and types of approaches to $\mathrm{VaR}$ estimation are growing exponentially and that it is impossible to take all of them into account; see also Duffie and Pan (1997) for a review.

When the returns of each portfolio asset are small, the portfolio return is given approximately by $r_{t} \approx \Sigma_{j} x_{j, t} r_{j, t}$ where $x_{j, t}$ for $j=1, \ldots, n$ are the portfolio weights of the $j^{\text {th }}$ asset at time $t$. The $100 \alpha \%$ one-day-ahead VaR denoted by $v_{\alpha, t}$ is defined by:

$$
P\left[r_{t} \leq v_{\alpha, t} \mid r_{j, t-i}, j=1, \ldots, n ; i=1, \ldots(t-1)\right]=\alpha
$$

For a two-asset portfolio with $x_{1, t}=x_{2, t}$ and an AR(1)-GARCH(1,1) model for both assets there are losses larger than $v_{\alpha, t}$ if:

$$
0.5 \sum_{j=1}^{2}\left\{c_{j}+\varphi_{j} r_{j, t-1}+\left(\alpha_{j}+\beta_{j} \varepsilon_{j, t-1}^{2}+\gamma_{j} \sigma_{j, t-1}^{2}\right)^{1 / 2} \eta_{j, t}\right\} \geq v_{\alpha, t}
$$

After estimating the whole model the $\mathrm{VaR} v_{\alpha, t}$ can be estimated by simulation if one is able to sample from the joint distribution of innovations, which is achieved easily by copula.

\section{B. Dependence in Financial Time Series}

Many financial series display several characteristics known as stylized facts. For the univariate series, the main stylized facts are: expected value of returns near zero, presence of autocorrelation in the square of returns, excess kurtosis, volatility clustering and long persistence. There are also 
deviations from multivariate normality. The conditional correlation between two variables $X$ and $Y$ at an exceedance level $\xi$ is defined as:

$$
\bar{\rho}(\xi)=\left\{\begin{array}{l}
\operatorname{corr}(X, Y \mid X>\xi, Y>\xi), \text { if } \xi \geq 0 \\
\operatorname{corr}(X, Y \mid X<\xi, Y<\xi), \text { if } \xi \leq 0
\end{array}\right.
$$

where corr denotes the linear correlation conditioned on the specified region. The concept of tail dependence relates to the amount of dependence in the upper-right-quadrant or lower-left-quadrant tail of a bivariate distribution. There is symmetric dependence when the amount of dependence in both quadrant is equal, being asymmetric otherwise.

Definition: A measure of the tail dependence is given by the coefficient of the upper and lower tail dependence. Let $(X, Y)$ be a bivariate continuous random variable with marginal distribution functions $F_{X}$ and $F_{Y}$. The coefficient of upper tail dependence of $(X, Y)$ is defined as:

$$
\lambda_{U}=\lim _{\alpha \rightarrow 1-} P\left\{Y>F_{Y}^{-1}(\alpha) \mid X>F_{X}^{-1}(\alpha)\right\},
$$

provided that the limit $\lambda_{U} \in[0,1]$ exists. If $\lambda_{U}>0, X$ and $Y$ are said to be asymptotically dependent in the upper tail. If $\lambda_{U}=0, X$ and $Y$ are said to be asymptotically independent in the upper tail. If the limit does not exist, the tail dependence is not defined. The coefficient of lower tail dependence, $\lambda_{L}$ is defined similarly.

Since $P\left\{Y>F_{Y}^{-1}(\alpha) \mid X>F_{X}^{-1}(\alpha)\right\}, \lambda_{U}$ can be written as a limit of:

$$
\frac{1-P\left\{X \leq F_{X}^{-1}(\alpha)\right\}-P\left\{Y \leq F_{Y}^{-1}(\alpha)\right\}+P\left\{X \leq F_{X}^{-1}(\alpha), Y \leq F_{Y}^{-1}(\alpha)\right\}}{1-P\left\{X \leq F_{X}^{-1}(\alpha)\right\}}
$$

Longin and Solnik (2001) studied the financial returns of pairs of stock indices of the five largest world markets (US, UK, France, Germany and Japan). They found that the correlation between large losses did not converge to zero, as expected in the bivariate normal case, but tended to increase when the level $\xi(\xi \leq 0)$ increased in absolute levels, whereas the correlation between large gains tended to decrease and converged to zero when the level $\xi$ increased. They also found evidence that the correlation between financial returns increased in bear markets, but did not increase in bull markets, i.e., there is asymmetric tail dependence. 
This complex dependence structure and fat tails are not adequately covered by traditional methods such as the variance-covariance one, the historical approach, or GARCH models. Copula and extreme value theory can be very helpful as claimed in this paper.

\section{Copula}

The notion of copula dates back to the early works of Hoeffding and Sklar. Nelsen (2006) presents a nice introduction to its theoretical and practical aspects. Let $\left(X_{1}, \ldots, X_{n}\right)$ be a $n$-dimension random vector with marginal distributions $F_{1}, \ldots, F_{n}$ and the associated uniform random variables $U_{i}=F_{i}\left(X_{i}\right), i=1, \ldots, n$. If $C\left(u_{1}, \ldots, u_{n}\right)$ is the distribution function of $\left(U_{1}, \ldots, U_{n}\right)$ then the distribution function of $\left(X_{1}, \ldots, X_{n}\right)$ is given by:

$$
H\left(x_{1}, \ldots, x_{n}\right)=C\left(F_{1}\left(x_{1}\right), \ldots, F_{n}\left(x_{n}\right)\right)
$$

and $C\left(u_{1}, \ldots, u_{n}\right)$ is the copula function. So copula can be interpreted as a function that links the marginal distributions of a random vector to form their joint distribution. Conversely, if $C$ is a copula and $F_{i}, i=1, \ldots, n$ are distribution functions, then the function $H$ defined by (6) is a joint distribution function with marginals $F_{i}, i=1, \ldots, n$. This shows the usefulness of copula in multivariate financial modeling. Patton (2006b) extended Sklar's theorem for the conditional case.

Sklar's theorem for conditional distributions: Let $F_{i}$ be the distribution of $X_{i}$ conditioned on $\boldsymbol{Z}$ (i.e., $X_{i} \mid \boldsymbol{Z}$ ), for $i=1, \ldots, n$ and let $H$ be the joint conditional distribution of $\boldsymbol{X} \mid \boldsymbol{Z}$. Then, there exists an $n$-dimensional copula $C$ such that for all $\boldsymbol{x} \in \Re^{n}$ and for any $\boldsymbol{z} \in \Lambda$

$$
H\left(x_{1}, \ldots, x_{n} \mid \mathbf{z}\right)=C\left(F_{1}\left(x_{1} \mid \mathbf{z}\right), \ldots, F_{n}\left(x_{n} \mid \mathbf{z}\right)\right) .
$$

If $F_{1}, F_{2}, \ldots, F_{n}$ are continuous then $C$ is unique. Conversely, if $C$ is an $n$-dimensional copula and $F_{i}$ is the conditional distribution function of $X_{i} \mid Z$, for $i=1, \ldots, n$, then the function $H$ defined by (7) is a conditional joint distribution function. An interesting feature of copula is that it is possible to simulate from the joint distribution $H$ using the marginal distributions and the copula functions.

For continuous random variable $X$ and $Y$ it can be shown that tail dependence is a copula property and hence the amount of tail dependence is invariant under strictly increasing transformations of $X$ and $Y$. An alternative definition of $\lambda_{U}$ and $\lambda_{L}$, using equation (5) (see, for instance Joe [1997], p 33) is: 


$$
\lambda_{U}=\lim _{u \rightarrow 1-} \frac{1-2 u+C(u, u)}{1-u}, \lambda_{L} \lim _{u \rightarrow 0+} \frac{C(u, u)}{u}
$$

where $C$ is a copula of the variables $X$ and $Y$. It shows that tail dependence is a pure copula property since it is independent of the margins of $X$ and $Y$. Most of the copula functions (e.g., the elliptical copula) have symmetric dependence. Some of them (e.g., like the normal copula) have lower and upper tail dependence equal to zero.

A copula $C$ which satisfies $C\left(\mathbf{u}^{a}\right)=[C(\mathbf{u})]^{a}$ for any exponent $a>0$, where $\mathbf{u}^{a}=\left(u_{1}, \ldots, u_{n}\right)^{a}=\left(u_{1}^{a}, \ldots, u_{n}^{a}\right)$ is called an extreme value copula. Two such copulae are the Gumbel and Gumbel-A copulae, which are discussed in this paper. The Gumbel copula is defined as:

$$
C\left(u_{1}, \ldots, u_{n}\right)=\exp \left\{-\left[\left(-\log u_{1}\right)^{1 / \theta}\right]^{\theta}\right\} .
$$

The parameter $0<\theta \leq 1$ controls the dependence between the random variables. $\theta=1$ implies independence. In the limit when $\theta \rightarrow+0$ there is perfect dependence. The lower tail dependence is zero, when $\theta=1$, and the upper tail dependence is $\left(2-2^{\theta}\right)$, when $\theta \rightarrow+0$.

The Gumbel-A copula is defined as:

$$
C_{\delta}(u, v)=u v \exp \left\{-\delta \frac{\log u \log v}{\log u v}\right\},
$$

with $0 \leq \delta \leq 1$, and we have independence when $\delta=0$. The lower tail dependence is equal to zero and the upper tail dependence is equal to $\delta / 2$. Observe that when $\delta=1$ the maximum upper tail dependence is 0.5 .

\section{A. Diagnostic and Empirical Copula}

The goodness-of-fit of copula models can be assessed by comparing the empirical and estimated copulae; see Junker, Szimayer and Wagner [2006]. The first tool is a graphical representation of the empirical and estimated copulae and the second one is a measure of distance.

Let $\chi$ be the sample $\chi=\left\{\left(x_{1}^{t}, \ldots, x_{n}^{t}\right)\right\}_{t=1}^{T}$. The empirical copula $\hat{C}$ is:

$$
\hat{C}\left(\frac{t_{1}}{T}, \frac{t_{2}}{T}, \ldots, \frac{t_{n}}{T}\right)=\frac{1}{T} \sum_{t=1}^{T} I_{\left[x_{1, t} \leq x_{1}^{\left(t_{1}\right)}, \ldots, x_{n, t} \leq x_{n}^{\left(t_{n}\right)}\right]},
$$

where $I$ is the indicator function $x_{n}^{\left(t_{j}\right)}, i=1, \ldots, n ; j=1, \ldots, n$ are the $t_{j}^{\text {th }}$ order statistics of the $i^{\text {th }}$ variable and $t_{1}, \ldots, t_{n} \varepsilon\{1, \ldots, T\}$. Therefore, the 
empirical copula is the proportion of elements from the sample that satisfies $x_{1, t} \leq x_{1}^{\left(t_{1}\right)}, \ldots, x_{n, t} \leq x_{n}^{\left(t_{n}\right)}$.

The distance between the empirical and a given estimated copula $C_{i}$ in a region $B$ of interest is defined as:

$$
d\left(\hat{C}, C_{i}\right)=\left[\sum_{\left(t_{1}, \ldots, t_{n}\right) \in B}\left(\hat{C}\left(\frac{t_{1}}{T}, \ldots, \frac{t_{n}}{T}\right)-C_{i}\left(\frac{t_{1}}{T}, \ldots, \frac{t_{n}}{T}\right)\right)^{2}\right]^{1 / 2}
$$

\section{B. Marginal Distribution of Innovations}

In order to model the joint distribution of innovations by copula it is necessary to choose the marginal distribution of innovations. Genest, Ghoudi and Rives (1995) used the empirical distribution, Dias and Embrechts (2004) used $t$-distribution, and Di Clemente and Romano (2005) used normal distribution in the center and GPD distribution in the tails. When estimating $\mathrm{VaR}$ it is interesting to use the GPD distribution in the left tail, which corresponds to large losses. In the other region, the right choice is not much relevant. Thus, this paper proposes the use of the $G P D$ distribution in the left tail and the empirical distributions otherwise.

The GPD is defined as:

$$
F_{W}(w)=\left\{1-(1+\vartheta \omega / \psi)^{-1 / \vartheta}\right\} I_{[\omega>0]}
$$

where $\psi>0$. It is considered that $\vartheta>0$, which corresponds to heavytailed distributions. For any $j^{\text {th }}$ series let $N_{j}\left(c_{j}\right)$ be the number of observations that exceeds a threshold value $c_{j}$ and denotes the excesses by $W_{j, i}, i=1, \ldots, N_{j}\left(c_{j}\right)$.

Assuming that $N_{j}\left(c_{j}\right)$ excess observations are independent identically distributed (iid) with exact GPD distribution, Smith (1987) showed that the $M L E$ of the $G P D$ parameters are consistent and asymptotically normal when $\vartheta>1 / 2$. Since in this case the support is $\omega \geq 0$ and the interest is in large losses, the GPD distribution is fitted to $\left\{\eta_{j, t}^{\prime}=-\eta_{j, t}, j\right.$ $=1, \ldots, k$, i.e., to the negative of the innovations. Notice that the GARCH innovations are iid.

\section{Model Estimation}

All the model parameters can be estimated by the maximum likelihood method, but this method may be computationally cumbersome. An alternative is the method of Inference Function for Margins (IFM) (see 
Joe and Xu [1996]), which estimates the model in two stages. In the first stage the marginal models are estimated independently, and in the second stage, the copula function is estimated conditioned on the first stage estimate. Under some regularity conditions Patton (2006a) showed that this estimator is also asymptotically normal. This approach has been widely used in the literature (e.g., Genest, Ghoudi and Rives [1995], Dias and Embrechts [2004], Di Clemente and Romano [2005] and Patton [2006a, 2006b]). In this paper the estimation is done as follows:

1. Fit ARMA-GARCH models for each return series. The residuals are the estimates of the innovations.

2 . Fit the GPD to the negative of each innovation series after choosing threshold values. McNeil and Frey (2000) used steps one and two in the estimation of univariate GARCH-EVT VaR measures.

3 . The copula function parameters are fitted to the residuals of step one with two marginal distributions. In the first case, one uses the empirical distribution of the residuals. In the second case, the GPD estimated in step two is used for the left tail, and the empirical distribution otherwise. A censorship type approach is proposed when estimating the copula function parameters, since the main interest is in large losses.

For the bivariate case, consider region $\mathrm{D}$ where none of the negatives of the marginal innovations are larger than some threshold values. These threshold values are not necessarily the same as the threshold values chosen for the GPD distributions. Since the main interest is modeling large losses there is no interest in the exact distribution in region D. Therefore, the observations in this region can be treated as censored and, in this case, the likelihood is given by:

$$
l(\Theta)=\left\{P_{\Theta}\left[\left(\eta_{1, t}^{\prime}, \eta_{2 t}^{\prime}\right) \in D\right]\right\}^{n_{1}} \prod_{D^{c}} c\left(F_{1}\left(\eta_{1, t}^{\prime}\right), F_{2}\left(\eta_{2, t}^{\prime}\right) ; \Theta\right)
$$

where $\Theta$ is a vector of copula parameters, $F_{1}$ and $F_{2}$ are the GPDs fitted to the residuals, $n_{1}$ is the number of observations in $\mathrm{D}$ and $c\left(u_{1}, u_{2}\right)$ is the density function associated with the distribution function $C\left(u_{1}, u_{2}\right)$.

\section{Application}

The proposed methodology is applied to a two-asset portfolio composed of IBOVESPA and MERVAL indices. The data cover the period from 3/3/1997 to 11/7/2000. IBOVESPA and MERVAL data were collected from www.ipea.gov.br and from www.bolsar.com, respectively. When at least one market was closed, this day was discarded, resulting in a total 

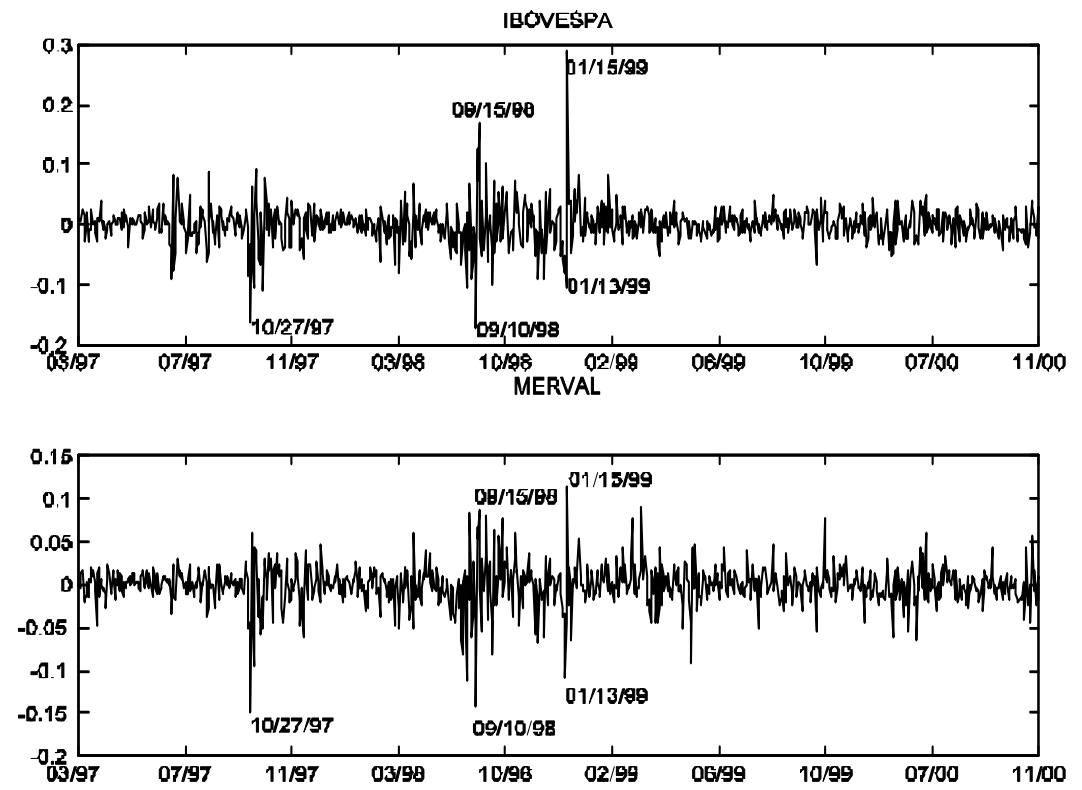

FIGURE 1- Returns of IBOVESPA and MERVAL from March 3rd, 1997 to November 7th, 2000.

of 900 observations (49 days discarded). For the latter series the excess kurtosis values were $(12.58,5.93)$, the asymmetries $(0.60,-0,62)$, minima $(-0.172,-0,148)$ and maxima $(0.288,0.116)$. The returns of both series are presented in figure 1.

First, models were selected for each marginal series. The ARMA model was not necessary for any series and GARCH(1,1) model was selected to explain the conditional volatility of both series. The estimated models are given in table 1 . The estimated persistence of both series is high, but the persistence of IBOVESPA (0.966) is larger than that of MERVAL (0.957). The estimated correlation function of the residuals and their squared series showed that the model was able to explain the level and the conditional volatility of the returns.

The estimates of the GPD distribution fitted to the largest $10 \%$ negative values of each residuals series are given in table 2. Both shape parameters are positive, corresponding to the heavy-tailed distribution, but the estimated parameter for IBOVESPA is much larger than that for MERVAL. The estimates of the copula parameters (with empirical standard deviations in brackets) are presented in table 3 for Gumbel-GPD (Gumbel copula with the marginal distribution fitted by GPD and empirical distribution), Gumbel-emp (Gumbel copula with empirical 
TABLE 1. Estimates of the $\operatorname{GARCH}(1,1)$ for the Marginal Return Series. Estimated Standard Deviation in Brackets.

\begin{tabular}{lccc}
\hline parameter & $\hat{\alpha}\left(10^{-4}\right)$ & $\hat{\beta}$ & $\hat{\gamma}$ \\
\hline IBOVESPA & 0.3751 & 0.2117 & 0.7539 \\
& $(0.0859)$ & $(0.0230)$ & $(0.0269)$ \\
MERVAL & 0.2842 & 0.1621 & 0.7952 \\
& $(0.0570)$ & $(0.0147)$ & $(0.0198)$ \\
\hline
\end{tabular}

TABLE 2. Estimates of the GPD Distributions with $10 \%$ of the Largest Losses. Estimated Standard Deviation in Brackets.

\begin{tabular}{lccc}
\hline parameter & $\hat{c}$ & $\hat{\vartheta}$ & $\hat{\psi}$ \\
\hline IBOVESPA & & 1.150 & 0.5422 \\
MERVAL & 1.217 & $(0.0140)$ & $(0.0101)$ \\
& 1.155 & 0.0150 & 0.8123 \\
& & $(0.0086)$ & $(0.0152)$ \\
\hline
\end{tabular}

TABLE 3. Estimates of the Copula Parameters and the Upper Tail Coefficient. Estimated Standard Deviation in Brackets.

\begin{tabular}{|c|c|c|c|c|}
\hline \multirow{2}{*}{$\begin{array}{l}\text { Marginal } \\
\text { distribution }\end{array}$} & \multicolumn{2}{|c|}{ Gumbel } & \multicolumn{2}{|c|}{ Gumbel-A } \\
\hline & $\hat{\theta}$ & $\hat{\lambda}_{U}$ & $\hat{\delta}$ & $\hat{\lambda}_{U}$ \\
\hline GPG & $\begin{array}{c}0.5300 \\
(0.0481)\end{array}$ & $\begin{array}{c}0.5562 \\
(0.0441)\end{array}$ & $\begin{array}{c}0.8232 \\
(0.0531)\end{array}$ & $\begin{array}{c}0.4742 \\
(0.0270)\end{array}$ \\
\hline Empirical & $\begin{array}{c}0.6011 \\
(0.0470)\end{array}$ & $\begin{array}{c}0.4120 \\
(0.0363)\end{array}$ & $\begin{array}{c}0.9581 \\
(0.0181)\end{array}$ & $\begin{array}{c}0.4790 \\
(0.0092)\end{array}$ \\
\hline
\end{tabular}

distribution for all the range of the residuals), Gumbel-A-GPD and Gumbel-A-emp. The first deciles of each marginal innovation series were used as threshold values for region D and for the GDP distributions. The table also presents the estimates of upper tail coefficients. The estimates for the upper tail coefficients for all models are around 0.5 showing an asymptotic upper tail dependence. There is no significant difference among the upper tail estimates at the 5\% level.

The isolines of the empirical and estimated Gumbel-GPD and Gumbel-emp models are presented in figure 2. In each plot, the largest losses are in the top right region of the figure. Figure 2a indicates a good agreement for the Gumbel-GPD for largest losses, but the fit is not good when the loss becomes smaller. On the other hand, the Gumbel-emp model (see figure $2 \mathrm{~b}$ ) presents a more balanced adjustment at all loss levels. These results are confirmed when the distance between the 

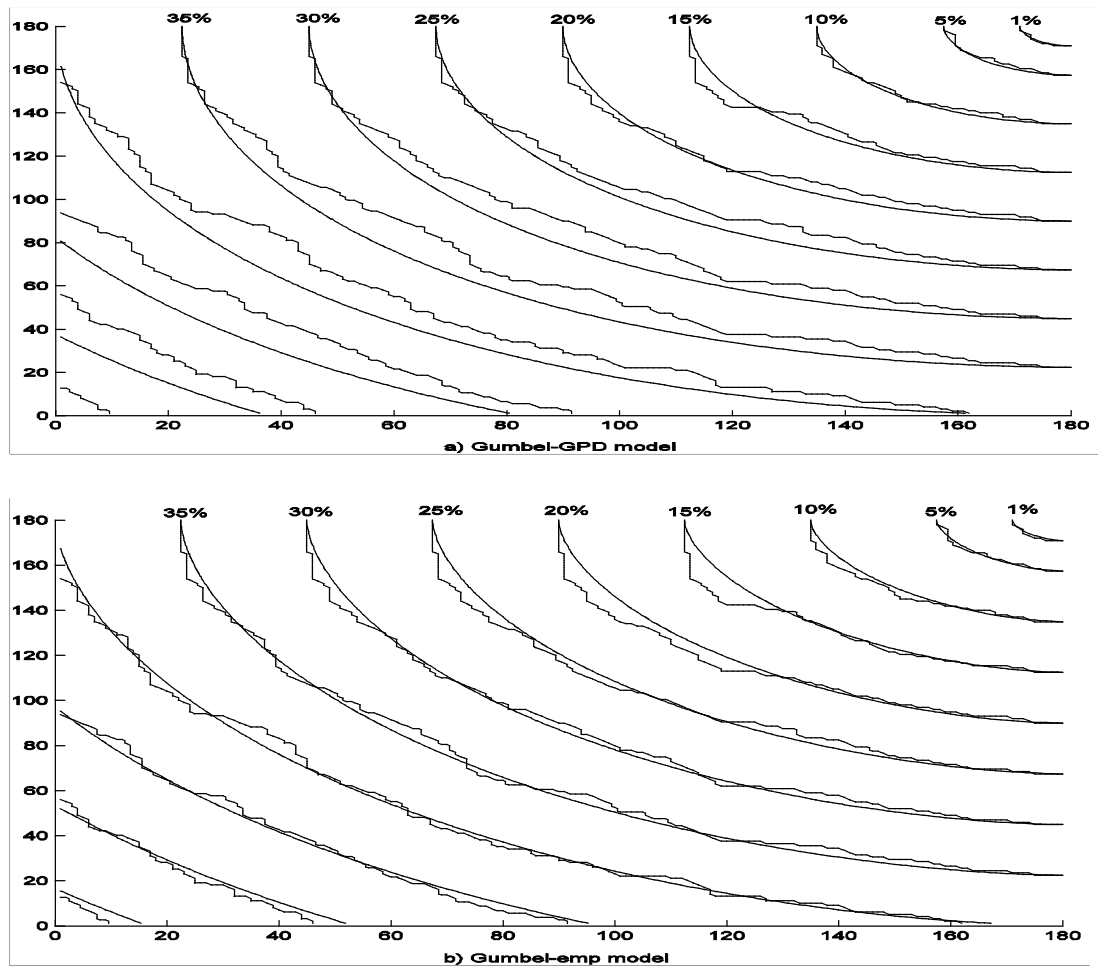

FIGURE 2-Isolines for Empirical and Estimated Copulae: (a)

Gumbel-GPD Model and (b) Gumbel-emp Model. Starting fom the Top Right 1\%, 5\%, 10\%, 15\%, ... Largest Losses.

empirical and the estimated copulae was used. These distances were evaluated only for the observations with the largest losses and are presented in table 4 for the $20 \%, 10 \%, 5 \%$ and $1 \%$ largest losses.

In order to compare the VaR estimated by the models, a backtest for the $95 \%$ and $99 \%$ Var estimates was employed. Suppose there is interest in estimating the VaR for the $t_{0}^{\text {th }}$ observation. First, the whole model is estimated using data up to the $\left(t_{0}-1\right)-t h$ observation. Then, $v_{a, t 0}$ is estimated by simulating the innovations from the estimated copula. This whole process can be repeated until the last observation and counted the number of cases where the observed return is smaller than the estimated $\mathrm{VaR}$. If the model is adequate it is expected to have a proportion equal to. In this exercise, the model was re-estimated only once in every 50 observations due to the computational cost of the procedure and because the estimates are not expected to have large modifications when only a 
TABLE 4. Distance Between Empirical and Estimated Copulae for the 20\%, $10 \%, 5 \%$ and $1 \%$ Largest Losses. Best Fit is Shown in Boldface.

\begin{tabular}{lllll}
\hline Copula model & $20 \%$ & $10 \%$ & $5 \%$ & $1 \%$ \\
Gumbel-GPD & 1.0500 & 0.2452 & 0.0899 & $\mathbf{0 . 0 0 4 1}$ \\
Gumbel-emp & $\mathbf{0 . 3 8 0 6}$ & $\mathbf{0 . 2 2 1 8}$ & $\mathbf{0 . 0 7 1 3}$ & 0.0056 \\
Gumbel-A-GPD & 1.1083 & 0.4365 & 0.1559 & 0.0079 \\
Gumbel-A-emp & 0.3856 & 0.2283 & 0.0738 & 0.0057 \\
\hline
\end{tabular}

TABLE 5. Proportion of Observations with Portfolio Loss Larger than the Estimated VaR Values with $(100(1-\alpha) \%)$ Confidence Intervals. Number of Observations is shown in Brackets. Best Performance shown in Boldface.

\begin{tabular}{|c|c|c|}
\hline \multirow[b]{2}{*}{ Models/nominal } & \multicolumn{2}{|c|}{$\alpha$} \\
\hline & 0.05 & 0.01 \\
\hline Gumbel-emp & $0.0798(36)$ & $0.0254(11)$ \\
\hline Gumbel-GPD & 0.0427 (19) & $0.0075(4)$ \\
\hline Gumbel-A-emp & $0.1225(55)$ & $0.0270(12)$ \\
\hline Gumbel-A-GPD & $0.0724(32)$ & $0.025(11)$ \\
\hline Bivariate GARCH (BEKK) & $0.0378(17)$ & $0.0067(3)$ \\
\hline Bivariate GARCH (DCC) & $0.0355(16)$ & $0.0067(3)$ \\
\hline Bivariate EWMA & $0.0378(17)$ & $0.0067(3)$ \\
\hline GARCH-n (Portfolio) & $0.0578(26)$ & $0.0178(8)$ \\
\hline GARCH-t (Portfolio) & $0.0622(28)$ & $0.0111(5)$ \\
\hline EWMA (Portfolio) & $0.0556(25)$ & $0.0200(9)$ \\
\hline Historical simulation (Portfolio) & $0.0178(8)$ & $0.0000(0)$ \\
\hline
\end{tabular}

fraction of the observations is modified. Observe that by using this approximation it is necessary to simulate from the copula only in every 50 observations. However, the VaR values are modified in every observation because the conditional mean and variance change (for instance, according to (1-3) for the $\operatorname{AR}(1) x \operatorname{GARCH}(1,1)$ model). The procedure was started with $t_{0}=451$ until $t_{0}=900$. A total of 450 observations were used to estimate the model, leaving out the first 50 observations in the last series when new 50 observations were added.

We also estimated the VaR by using different approaches. The VaR values were also estimated using three other bivariate approaches: Engle and Kroner (1995) first order BEKK model, the Engle (2002) Dynamic Conditional Correlation $(D C C)$ model and the Exponentially Weighted Moving Average (EWMA) technique. A univariate approach was also 
applied directly to the portfolio return series using $\operatorname{GARCH}(1,1)$ model with normal and $t$ distributions, EWMA, and historical simulation. All the GARCH models, univariate or bivariate, were fitted with a constant in the level equations. The univariate EWMA and historical simulation are very easy to implement. Thus, only in these cases, the models used for the $\mathrm{VaR}$ were estimated in every observation and not in every 50 observations. In the EWMA approaches, the weighting factor was selected by minimizing the mean square difference between the one-step estimation of the volatility and the square of the observed return. The proportion of observations with loss larger than the estimated $\mathrm{VaR}$ is presented in table 5 for $95 \%$ and $99 \%$ confidence intervals.

The analysis shows that the Gumbel-GPD model presented the best result with empirical proportion approximately equal to the nominal values for both confidence intervals. The copula models with empirical distribution tended to underestimate the VaR values, showing the importance of using heavy-tailed distributions. The other approaches, in general, have a bad performance, at least at one of confidence intervals. For instance, the GARCH-t $(1,1)$ model fitted directly to the portfolio returns had a good performance at the $99 \%$ confidence interval, but its performance was only reasonable at the $95 \%$ confidence interval. On the other hand, the EWMA approach had a good performance at the $95 \%$ confidence interval but not at the $99 \%$ confidence interval.

\section{Final Remarks}

The proposed model outperformed other traditional models. The Gumbel copula with the GPD marginal distribution produced a good fit in the negative tail. The estimation procedure showed that it clearly emphasized the fit in the tail with less attention to the other observations. The estimation procedure adopts a censorship-type approach. This approach also allows modeling data with asymmetric dependence with symmetric copula function, considerably enlarging the copula functions that can be used with this type of data. The procedure can also be used to model largest gains. Although the results clearly indicated that copula and extreme value theories can be very useful in estimating the $\mathrm{VaR}$ of a portfolio, further investigation is necessary. For instance, it is necessary to look for an efficient estimation algorithm, to test other copula functions, and to look at portfolios of a higher dimension. 


\section{References}

Di Clemente, A., and Romano, C. 2005. Measuring portfolio value-at-risk by a copula and EVT based approach. Studi Economici 85:29-57

Dias, A., and Embrechts, P. 2004. Dynamic copula models for multivariate high-frequency data in finance. Working Paper. Zurich: ETH.

Duffie, D., and Pan, J. 1997. An overview of value at risk. Journal of Derivatives (Spring): 7-49.

Engle, R. 2002. Dynamic conditional correlation - a simple class of multivariate GARCH. Journal of Business and Economics Statistics 20: 339-350.

Engle, R., and Kroner, K. F. 1995. Multivariate simultaneous generalized ARCH. Econometric Theory 11(1): 122-150.

Genest, C.; Ghoudi, K.; and Rives, L.-P. September 1995. A semiparametric estimation procedure of dependence parameters in multivariate families of distributions. Biometrika 82: 543-552.

Joe, H. 1997. Multivariate Models and Dependence Concepts. London: Chapman and Hall.

Joe, H., and Xu, J. J. 1996. The estimation method of inference functions for margins for multivariate models. Technical Report 166. Vancouver: University of British Columbia, Department of Statistics.

Junker, M.; Szimayer, A.; and Wagner, N. 2006. Nonlinear term structure dependence: Copula functions, empirics, and risk implications. Journal of Banking and Finance 30 (April): 1171-1199.

Longin, F., and Solnik, B. 2001. Extreme correlation of international equity markets. Journal of Finance 56 (April): 649-676.

Manganelli, S., and Engle, R.F. 2004. A comparison of Value at Risk in Finance. Chapter 9. In G. Szegö (ed.). Risk Measures for the $21^{\text {st }}$ Century. Wiley Finance Series.

McNeil, A., and Frey, R. 2000. Estimation of tail-related risk measures for heteroscedastic financial time series: An extreme value approach. Journal of Empirical Finance 7 (November): 271-300.

Nelson, D. B., and Cao, C. Q. 1992. Inequality constraints in the univariate GARCH model. Journal of Business and Economic Statistics 10 (April): 229-235.

Nelsen, R. B. 2006. Introduction to Copulas. $2^{\text {nd }}$ Ed., NY: Springer Verlag.

Patton, A. 2006a. Estimation of multivariate models for time series of possibly different lengths. Journal of Applied Econometrics 21(2): 147-173.

Patton, A. 2006b. Modelling asymmetric exchange rate dependence. International Economic Review 47(2): 527-556.

Seymour, A. J., and Polakow, D. A. 2003. A coupling of extreme-value theory and volatility updating with Value-at-Risk estimation in emerging markets: A South African test. Multinational Finance Journal 7 (March/June): 3-23.

Smith, R. L. 1987 Estimating tails of probability distributions. The Annals of Statistics 15 (3): 1174-1207. 